\title{
Revisiones
}

"El riesgo mayor para la salud humana derivado de la presencia del mercurio en la naturaleza se centra en la exposición ocupacional a este metal".

OMS

\section{Intoxicación ocupacional por mercurio}

\author{
Mercury occupational poisoning
}

\author{
Augusto V Ramírez ${ }^{1}$ \\ ${ }^{1}$ Médico del Trabajo. American College of Occupational and Environmental Medicine.
}

\begin{abstract}
Resumen
El mercurio, metal pesado ampliamente utilizado por el hombre, es muy tóxico; produce daño al sistema nervioso central, perturbaciones del comportamiento y lesiones renales. Se acumula en todos los seres vivos y no es esencial para ningún proceso biológico. La toxicidad del mercurio está directamente relacionada con su estado químico. El metilmercurio es la forma más dañina, con efectos neurotóxicos en adultos y en fetos de madres expuestas. El mercurio metálico no es menos tóxico. Las sales de mercurio inorgánico afectan directamente al riñón. Clínicamente, en la exposición ocupacional a mercurio se encuentra la triada clásica: temblor, alteración de la personalidad y estomatitis. En los últimos años se ha demostrado también alteración en la visión cromática. La exposición aguda se evalúa midiendo el mercurio en la sangre, mientras que la exposición crónica y ocupacional se determina mejor dosándolo en orina homogenizada de 24 horas. Los quelantes del metal -BAL, sus derivados o la D-penicilamina- son usados para tratar la intoxicación aguda o crónica. Palabras clave: Intoxicación por mercurio; toxicología; exposición ocupacional.
\end{abstract}

\begin{abstract}
Mercury, heavy metal widely used, is very toxic and produces protoplasmic damage to central nervous system, disturbances of behaviour and renal injuries. Mercury is accumulated in all living beings and is not essential for any biological process. Mercury toxicity is directly related to its chemical state. Metil-mercury is the most harmful form with neurotoxic effects in adults and foetuses of exposed mothers. Metallic mercury is not less toxic. Inorganic mercury salts affect mainly the kidney. In occupational exposure to mercury, we can find the classic triad: tremor, personality alterations and stomatitis. In the last years alteration in the chromatic vision has also been demonstrated. We use mercury blood measurement to evaluate acute mercury exposure. Occupational exposure is better determined by measuring composite 24 hours urine. Chelating agents -BAL or D penicillamine- are used to treat both acute and occupational poisoning.
\end{abstract}

Key words: Mercury poisoning; toxicology; occupational exposure.

\section{INTRODUCCIÓN}

El mercurio $(\mathrm{Hg})$ es un metal blancoplateado muy tóxico, el único en estado líquido a $0^{\circ} \mathrm{C}$, muy denso y poco compresible; de tensión superficial muy alta y débil reacción calorífica, posee gran capacidad de amalgamar a casi todos los metales. Se evapora a $13^{\circ} \mathrm{C}$ y encontramos trazas de él en cualquier producto que se analice. No es esencial para ningún proceso biológico, pero se acumula en la mayoría de seres vivos. En la naturaleza existe como sulfuros de mercurio (cinabrio, rojo), de arsénico (rejalgar), hierro (piritas), mixto (metacinabrio, negro), de antimonio (estibina), pero también se le halla directamente unido a minerales de zinc, cobre, oro y plomo ${ }^{(1)}$.

Aparte del estado natural, existe un importante aporte antropogénico proveniente de su metalurgia, de sus aplicaciones en industrias diversas y de las aguas residuales de las ciudades. Se calcula que cada año mil toneladas son liberadas desde redes de alcantarillado a la superficie de la tierra ${ }^{(2)}$.
La producción de mercurio se cuantifica en unidades llamadas 'frascos' (por el frasco estándar usado en esta industria, que es de hierro y que sirve de medida: 2,5 litros y $34,5 \mathrm{~kg}$ de peso). Los principales países productores son España: Almadén (7 500 000), Eslovenia: Idria (3 000 000), Italia: Monte Amiata (2 000 000), Perú: Huancavelica (1 500 000), EE UU: New Almaden (1 000 $000)$, New Idria (600 000) y McDermitt (400 000 frascos) (2).

Actualmente, solo la mina de Almadén se encuentra en producción. Las minas de Huancavelica, explotadas desde la colonia, fueron las mayores productoras de intoxicaciones laborales por este metal en el Perú.

El $50 \%$ de la producción mundial de mercurio se usa en la fabricación de lámparas de vapor, tubos fluorescentes, termómetros clínicos e industriales y otros instrumentos, como barómetros, manómetros, esfingomanómetros, lentes de telescopios, lámparas de difusión y ultravioleta, conmutadores, cátodos electrolíticos, turbinas de vapor. Otras industrias importantes son: metalurgia del oro y plata, fabricación de pilas y baterías, amalgamas dentales, biocidas, fungicidas, pesticidas y productos farmacéuticos. En cada una de estas industrias existe riesgo de exposición ocupacional. Sin embargo, desde la segunda mitad del siglo XX, el uso del mercurio se ha ido dejando de lado por las graves intoxicaciones que produce y por la aparición de tecnología que va desplazándolo, pero aún su concentración en combustibles fósiles, carbón y petróleo es elevada ${ }^{(3,4)}$.

\section{TOXICIDAD}

La toxicidad del mercurio se conoce desde antiguo, por Hipócrates, Plinio y Galeno. Las primeras descripciones de los efectos tóxicos de sus vapores como riesgo laboral fueron descritos por Ellenberg en Von der Grifftigen Bensen Terupffen von Reiichen der metal (1473). Posteriormente, escritos como los de Paracelso (1533) y los de B. Ramazzini -en De Morbis Artificium Dia- 
triba (1700)- describen el cuadro clínico de intoxicación ocupacional. Los incas usaron como pintura el cinabrio, mineral del que se extrae el mercurio; lo llamaron llampi.

No existe unanimidad en cuanto al umbral medio de toxicidad humana; la investigación actual en salud ha establecido los límites de toxicidad del mercurio entre 50 y $160 \mu \mathrm{g} /$ día $^{(1,5)}$.

\section{TOXICOCINÉTICA}

El ingreso del mercurio es por las vías respiratoria, digestiva y cutánea.

La vía respiratoria es por inhalación. En salud ocupacional esta vía es la más importante y, tanto el mercurio elemental como el inorgánico y sus compuestos, puede ingresar por inhalación y alcanzar la sangre con una eficiencia del $80 \%$.

La vía digestiva es por ingestión. En el tracto gastrointestinal, el mercurio inorgánico se absorbe en cantidad menor al $0,01 \%$, probablemente por su incapacidad de reacción con moléculas biológicamente importantes, al formar macromoléculas que dificultan su absorción y porque pasa por un proceso de oxidación. Los compuestos inorgánicos de mercurio (sales) se absorben entre 2 y $15 \%$, dependiendo de su solubilidad. Mientras que, en contraste, la absorción de los compuestos orgánicos por esta vía es $95 \%$, independiente de si el radical metilo está unido a una proteína o no.

La vía cutánea es por contacto. Se ha descrito casos de intoxicación por aplicación tópica de compuestos que contenían metilmercurio. Sin embargo, no está demostrado que esta vía tenga un papel importante en la exposición ocupacional, comparada con las otras. Es más, es posible que en el caso de aplicación de pomadas, el tóxico penetre en el organismo por inhalación, a partir del ungüento puesto en la piel, más que atravesándola directamente.

En relación al transporte y distribución, absorbido el mercurio es transportado por la sangre en un ratio glóbulo rojo/plasma entre 1,5 a 3. Para sus sales inorgánicas, esta relación es menor: 0,4. En general, el 90\% de los compuestos orgánicos se transporta en las células rojas, mientras que $50 \%$ del mercurio inorgánico es transportado unido a la albúmina. Como norma, a partir de la sangre su distribución en el organismo tiende a alcanzar un estado de equilibrio dinámico determinado por dosis, duración de la exposición, grado de oxidación, concentración de sus compuestos en la sangre, concentración en relación con grupos sulfi- drilos libres, afinidad con los componentes celulares y velocidad de asociación/disociación del complejo mercurio-proteína.

Sin embargo, cabe destacar su gran afinidad por el encéfalo, quizá porque la mayor parte del mercurio circulante va al cerebro, más que a hígado o riñón. En el encéfalo, tiene mayor afinidad por la sustancia gris que por la blanca. Los niveles más altos de mercurio son hallados en ciertos grupos neuronales del cerebelo, médula espinal, pedúnculos y mesencéfalo, aunque también se le ha detectado en epitelio de tiroides y páncreas, en células medulares de las glándulas adrenales, en espermatozoitos, epidermis y cristalino.

Se estima que el contenido normal de mercurio en el organismo humano oscila entre 1 y 13 miligramos, del cual $10 \%$ es metilmercurio. Su distribución en el organismo es: músculo 44 a $54 \%$, hígado $22 \%$, riñón $9 \%$, sangre 9 a $15 \%$, piel $8 \%$, cerebro 4 a $7 \%$ e intestino $3 \%{ }^{(6)}$.

La biotransformación del mercurio se realiza por cuatro vías ${ }^{(7)}$ :

a. Por oxidación del vapor de mercurio metálico a mercurio divalente: La oxidación, mediada por la hidrógeno peróxido-catalasa en los peroxisomas, determina el tiempo de permanencia del vapor inhalado (crucial para alcanzar sitios sensibles), al disminuir su liposolubilidad y por tanto su toxicidad, pero la tendencia a la bioacumulación aumenta cuando esta oxidación se realiza en los tejidos.

El mercurio tiene gran afinidad por los grupos -SH de las proteínas. Éstos son tan abundantes que solo le permiten una breve presencia en estado iónico. El mercurio se une también a grupos fosforilos, carboxilo, amida y amina.

b. Por reducción del mercurio divalente a mercurio metálico: la reducción es mediada el sistema xantina oxidasa. Se ha demostrado el proceso contrario en animales de experimentación (rata, ratón) y en humanos.

c. Por metilación del mercurio inorgánico: Se ha demostrado la metilación de mercurio inorgánico en ratas, pero solo entre 0,05 y $0,26 \%$ de la dosis administrada. Se desconoce el lugar exacto de esta metilación, aunque se supone pueda ser el hígado. La metilación no ha sido demostrada en humanos.

d. Por conversión del metilmercurio en mercurio inorgánico. En la exposición laboral crónica se conoce el proceso de biodesmetilación en varios tejidos, pero es en el hígado donde se realiza en mayor proporción.

Con respecto al modelo toxicocinético de eliminación, la eliminación del tóxico se realiza desde los compartimientos central, periférico y el 'cuarto compartimiento'.

El compartimento central está formado por todos los órganos, menos riñón e hígado.

El compartimento periférico está constituido por el riñón, que acumula $\mathrm{Hg}$ por mayor tiempo y lo aclara muy lentamente, y por el hígado, que también lo acumula, pero por periodos cortos, pues lo aclara rápidamente. En este compartimento periférico se incluye los procesos de filtración glomerular, secreción biliar y secreción por la mucosa intestinal.

El 'cuarto compartimento' es el depósito per se y es el punto final antes de su excreción; lo integran orina, heces, pelo y uñas.

Si consideramos al organismo humano un modelo mono-compartimental abierto, la vida media del mercurio en exposición aguda es de 1,3 días y en exposición ocupacional continua, 36,5 días. En exposición ocupacional, la vida media de los compuestos inorgánicos de mercurio es de 40 días. La cantidad de mercurio excretada por vía renal/heces es entre 50 y $55 \%$ de la dosis total absorbida; por saliva equivale al $25 \%$ de la concentración sanguínea y al 10\% de la urinaria; por sudor es $15 \%$, suficiente para tenerla en cuenta en el balance global; y la vía respiratoria, por exhalación, interviene hasta con $7 \%^{(5,7)}$ (Figura 1).

\section{TOXICODINÁMICA}

Los efectos tóxicos del mercurio, inorgánico y orgánico, son debidos a que en su forma iónica no establece enlaces químicos.

Al revisar la acción sobre los sistemas enzimáticos, el mercurio es tóxico, porque precipita las proteínas sintetizadas por la célula, principalmente las neuronas, y porque inhibe los grupos sulfidrilo de varias enzimas esenciales. En estado iónico, se fija a los grupos celulares ricos en radicales - SH, altera varios sistemas metabólicos y enzimáticos de la célula y su pared e inhibe la síntesis de proteínas en la mitocondria, afectando su función energética. En el riñón disminuye la actividad de las fosfatasas alcalinas de los túbulos proximales y altera el transporte de potasio y la ATP-asa en la membrana. En el encéfalo, la neurona de cerebro y cerebelo es la parte más sensible. En el sistema enzimático, inhibe enzimas esenciales: catalasas plasmáticas, colineste- 
rasa globular, glutation-reductasa globular, glutation-reductasa cerebral, galactoxidasa, dopa-decarboxilasa, monoamino-oxidasa, glicero fosfatasa, succino-deshidrogenasa, di y trifosfo-piridín-nucleótido. Por todo esto, el mercurio puede causar lesión celular en cualquier tejido donde se acumule en concentración suficiente.

En varios órganos, incluido el riñón, y al igual que cadmio, cobre y zinc, el mercurio induce la formación de metalotioneína, un receptor proteico de peso molecular bajo, y se une a ella, saturando sus propios receptores. Cuando por la gran cantidad de tóxico presente la metalotioneína se forma en exceso, causa alteraciones orgánicas en el mismo sitio de su producción ${ }^{(8)}$.

\section{MERCURIALISMO OCUPACIONAL}

El diagnóstico de mercurialismo ocupacional en nuestro país no es frecuente, pero la sola presencia del metal en una actividad industrial debería ser suficiente para considerarlo en el diagnóstico diferencial con alguna otra intoxicación industrial. La industria minera formal maneja programas específicos de gestión para el control del riesgo en sus trabajadores. No sucede lo mismo en la pequeña y mediana industria aurífera de los ríos amazónicos, donde se extrae oro adicionando mercurio (azogue) a la grava, lo que condiciona riesgo no solo para los trabajadores, muchas veces niños, sino también para poblaciones ribereñas ${ }^{\left({ }^{9}\right)}$.

El diagnóstico cierto de mercurialismo ocupacional pasa por considerar los antecedentes de trabajo y lugar de procedencia, los factores determinantes de toxicidad, el estado físico y tipo de compuesto mercurial y la vía de ingreso.

Con relación a los antecedentes de trabajo y lugar de procedencia, como en toda enfermedad ocupacional, un diagnóstico preciso debe establecer relación causa-efecto. Por tanto, para que exista mercurialismo consecuente al trabajo tiene que haber necesariamente antecedente de exposición ocupacional. En cuanto a procedencia del trabajador, considerar que el mercurio se puede encontrar en muchas industrias, pero principalmente está presente en minería de oro, artesanal en los causes de los ríos o en la gran minería aurífera donde aparece como subproducto.

Los factores determinantes de toxicidad incluyen el estado fisicoquímico y la vía

\section{INTOXICACIÓN CRÓNICA OCUPACIONAL}

La exposición a mercurio asociada a malas prácticas de higiene laboral favorece el desarrollo de la intoxicación ocupacional, que se manifiesta por el cuadro clínico denominado mercurialismo o hidrargirismo ${ }^{(1,2,4-7-7,9,10)}$, que tiene características propias de acuerdo a su fase toxicocinética.

En la fase de absorción o impregnación, los síntomas son generales $\mathrm{e}$ inespecíficos: pérdida de apetito, adelgazamiento, cansancio fácil, cefalea, mareos, insomnio, artralgias y parestesias.

En la fase de intoxicación, encontramos ya el cuadro patognomónico, con los siguientes síndromes:

-Síndrome digestivo: caracterizado por sabor metálico, mal aliento, náuseas, vómitos y diarrea. En muy pocos casos aún se puede ver el estigma mercurial en los dientes, coloración pardusca en los incisivos

de ingreso del tóxico al organismo, idiosincrasia individual, tasas de excreción y efectos sinérgicos o antagónicos con otros agentes.

Las propiedades e interacciones biológicas del mercurio varían para cada estado fisicoquímico y cada uno tiene propiedades toxicológicas diferentes. Así, el mercurio elemental (e-Hg; $\left.\mathrm{Hg}^{0}\right)$ es soluble en lípidos, difusible por las biomembranas y bioxidado intracelularmente a mercurio inorgánico. Las sales de mercurio inorgánico $\left(\mathrm{i}-\mathrm{Hg} ; \mathrm{Hg}^{2+}\right)$ son solubles en agua, pero menos difusibles por las biomembranas. $\mathrm{El} \mathrm{i-Hg} \mathrm{induce} \mathrm{la}$ síntesis de metalotioneina. Los compuestos alquil-mercúricos ( $\mathrm{Hg}-\mathrm{C}: \mathrm{me}-\mathrm{Hg}$ y al-Hg) son solubles en lípidos, altamente difusibles a través de las biomembranas y transformados muy lentamente a $\mathrm{i}-\mathrm{Hg}$. De otro lado, los compuestos orto- $\mathrm{Hg}$ y alox-Hg, a pesar de también ser solubles en los lípidos, en el organismo humano son rápidamente degradados a i-Hg.

Con respecto a la vía de ingreso, en salud ocupacional la más importante para el mercurio es la respiratoria, sin desdeñar las otras ${ }^{(1-3)}$. (diente de Letuelle), casi siempre asociado a pésima higiene bucal.

- Síndrome neurológico: Constituye el cuadro clásico del mercurialismo, antes llamado 'eretismo mercurial'. En una primera fase, se evidencia por irritabilidad, tristeza, ansiedad, insomnio, sueño agitado, temor, debilidad muscular, pérdida de memoria, excesiva timidez, susceptibilidad emocional, hiperexcitabilidad o depresión producidos por daño en los centros corticales del sistema nervioso central, que puede llevar a encefalitis, condicionante del síndrome psicoorgánico crónico y definitivo, que termina en la demencia del trabajador. La descripción del personaje del sombrerero loco de Lewis Carrol es la mejor visión profana del eretismo mercurial ${ }^{(11)}$.

El signo capital descrito desde antiguo es el temblor intencional, con características de temblor cerebeloso, asociado a ataxia, adiadococinesia y marcha cerebelosa, que hacen la diferencia con el de Parkinson. El temblor guarda relación con la gravedad de la intoxicación y con la concentración de mercurio en los tejidos. Se puede hallar exageración 
de los reflejos patelares, pero no son frecuentes espasmos musculares ni parálisis flácida. Histológicamente, se encuentra degeneración axonal y alteraciones en los paquetes sensitivos y motores.

- Síndrome renal: Se ha descrito lesión glomerular de varios tipos, desde lesión mínima de aspecto semejante a la de nefrosis lipoide, hasta glomerulonefritis proliferativa extracapilar, con proliferación del epitelio de la cápsula de Bowman, y glomérulonefritis extramembranosa. Se afirma unánimemente que el sistema inmunitario es el primer órgano blanco y que solo posteriormente aparece daño renal.

- Síndrome oftalmológico: Como signo precoz de intoxicación se describe casos aislados de escotomas anulares y centrales y visión tubular (restricción concéntrica de los campos visuales). Puede haber nistagmus. Al examen con lámpara de hendidura, y también como signo temprano de intoxicación, se puede encontrar el signo de Atkinson, reflejo parduzco bilateral y simétrico en la cápsula anterior del cristalino, que no afecta la visión. Estudios actuales apoyan el hecho que la exposición a vapores de mercurio induce un cuadro sub clínico de daño en la visión de colores, inclusive en lugares de trabajo, con indicadores de exposición menor al límite actual, lo que nos permite dudar de la protección real de ese límite en lo referido a efectos del mercurio sobre la visión ${ }^{(12-14)}$.

Otras alteraciones encontradas son las siguientes:

- Piel: Dermatitis de contacto localizada en manos, antebrazos o cara y lesiones hiperqueratósicas que pueden ulcerarse; y, en exposición crónica, alopecia reversible.

- Rinitis y conjuntivitis causadas por acción irritativa directa del mercurio.

- Sangre: Específicamente el cloruro de mercurio contenido en algunos antisépticos incrementa el colesterol, por lo que al exponerse a este compuesto puede aumentar el riesgo ateromatoso en patología preexistente de aorta.

- Hipersensibilidad: En exposición a sales de mercurio inorgánico (mercurioso o mercúrico) o al fenilmercurio se puede encontrar acrodinia, reacción de hipersensibilidad caracterizada por descamación, color rosado de las mejillas y plantas de los pies y manos, fotofobia, sudoración, irritabilidad e insomnio.

- Efectos teratógenos y cancerígenos: la exposición a mercurio elemental o a compuestos inorgánicos no produce cáncer ni teratogenicidad, que sí están demostrados en los compuestos orgánicos (metilmercurio) ${ }^{(15,16)}$.

La intoxicación mercurial ocupacional no necesariamente es causada por exposición a cantidades elevadas de mercurio en el ambiente laboral, sino que también puede ocurrir con niveles bajos de exposición $(1,2)$.

\section{MERCURIO ORGÁNICO}

Es la forma química de mercurio unida al carbono. Existen numerosos compuestos orgánicos, pero los principales son: alquilos, arilos y alcoxialquilos, que son usados como pesticidas y son fuente importante de exposición en agricultura. El mercurio orgánico es muy dañino para el sistema neurológico, pues al precipitar las proteínas afecta el sistema de transporte microtubular de la neurona.

Los compuestos organomercuriales se absorben fácilmente por ingestión y se excretan principalmente con las heces y no por el riñón ${ }^{(16)}$.

Aquí una digresión, los compuestos orgánicos del mercurio son importante preocupación en Salud Pública, pues a dosis potencialmente tóxicas se les halla en los músculos de los grandes peces condrictios (cartilaginosos): tiburón, pez espada, merlines y tollos; y en algunas especies de teleósteos: atunes (común, rojo, patudo, etc.), albacora, bonito del norte y en los mariscos.

Otro gran aporte de mercurio a la población general es el proveniente de las aún omnipresentes amalgamas dentales.

La OMS considera como valor de mercurio 'normal' en sangre $<10 \mu \mathrm{g} / \mathrm{L}$ y orina $<20 \mu \mathrm{g} / \mathrm{L}^{(17-19)}$.

En industrias que utilicen compuestos orgánicos, tener en cuenta que éstos tienden a descomponerse y a liberar vapores de metálicos, por lo que el control del ambiente laboral debe dirigirse hacia ambas formas químicas ${ }^{(1)}$.

\section{INDICADORES BIOLÓGICOS DE EXPOSICIÓN}

La legislación peruana norma la exposición a mercurio en el trabajo, remitiéndonos a criterios internacionales ${ }^{(20)}$. Entre otros, la ACGIH (Conferencia Gubernamental Americana de Higienistas Industriales) establece el valor de los indicadores biológicos de exposición (BEIs, por sus siglas inglesas) en trabajadores expuestos: 1) mer- curio inorgánico total en muestra de orina tomada antes de la jornada laboral: $35 \mu \mathrm{g} / \mathrm{g}$ creatinina; y, 2) mercurio inorgánico total en muestra de sangre tomada al final de la jornada diaria o al fin de semana laboral: $15 \mu \mathrm{g} / \mathrm{L}^{(21,22)}$.

En exposición ocupacional, se recomienda dosar mercurio en orina de 24 horas; $y$, en exposición aguda accidental, preferir su dosaje en sangre ${ }^{(23,24)}$.

En todos los casos, un programa de gestión de salud ocupacional para el riesgo mercurio debe iniciarse con su valoración en el ambiente de labor. Para este caso, el valor de referencia, valor umbral límite (TLV) de la ACGIH para mercurio elemental y sus formas inorgánicas es 0,025 $\mathrm{mg} / \mathrm{m}^{3} / 8$ horas/día/5 días semana ${ }^{(21,25)}$.

\section{TRATAMIENTO FARMACOLÓGICO}

Para el tratamiento farmacológico del mercurialismo se recomienda:

A. Para el mercurio inorgánico elemental:

- DMPS: (2,3 dimercaptopropano-1-sul-

fonato). Dimanal ${ }^{R}$ solución EV 5\%: 5 $\mathrm{mg} / \mathrm{kg}$, cada 6 horas las primeras 24 horas; el segundo día, cada 8 horas; y una dosis diaria en los días subsiguientes, hasta bajar el BEI al 50\%. Si no baja, continuar por vía oral a dosis de $100 \mathrm{mg}$ bid, 24 días más ${ }^{(5,26)}$.

- DMSA: (ácido 2,3 dimercaptosuccinico). Succimer ${ }^{R}$ vía oral, $30 \mathrm{mg} / \mathrm{kg}$ por día, durante 5 días, seguidos de $20 \mathrm{mg} / \mathrm{kg} / \mathrm{día}$, hasta 14 días ${ }^{(5)}$.

- BAL: (British Anti Lewisite) (2,3 - dimercaptopropanol). Solución oleosa al $10 \%$. Ampolla de $5 \mathrm{~mL}$ vía intramuscular profunda, con aguja 18 de 2" mínimo: 2,5 $\mathrm{mg} / \mathrm{kg}$ por dosis $(2,5 \mathrm{~mL} / \mathrm{kilo})$. Primer día: 1 dosis cada 4 horas. Segundo y tercer días: 1 dosis cada 6 horas. Días 4 al 10: 1 dosis cada 8 horas ${ }^{(5)}$.

- D-penicilamina. Vía oral y después de las comidas 15 a $40 \mathrm{mg} / \mathrm{kg}$ por dosis. No exceder $1-2 \mathrm{~g} / \mathrm{día}^{(5)}$. El uso de derivados penicilamínicos sigue siendo discutible, por las reacciones secundarias que conlleva: hipersensibilidad, problemas digestivos (náuseas, vómitos, alteraciones del gusto), dermatitis, alopecia, alteraciones hemáticas (leucopenia, trombocitopenia), alteraciones urinarias (proteinuria) y neurológicas, que agravan el cuadro de mercurialismo ${ }^{(27,28)}$.

B. Para el metilmercurio y otros compuestos orgánicos.

En intoxicación ocupacional por compuestos mercuriales orgánicos, se contraindica el uso de BAL, porque potencia 
la acción tóxica del mercurio. En este caso, se indica $\mathrm{N}$-acetil-DL-penicilamina, a dosis de $2-4 \mathrm{~g} /$ día. Si se presentase insuficiencia renal, el tratamiento será administrar ácido 2-3-dimercaptosuccinico y L-cisteína a grandes dosis, complementando con hemodiálisis. Repetir hasta la remisión de los síntomas ${ }^{(1,5)}$.

En el caso particular de quemadura por fulminato de mercurio, usar fomentos de tiosulfato de sodio al $10 \%$. Para conjuntivitis: colirio de tiosulfato al $2 \%{ }^{(2)}$.

\section{DIAGNÓSTICO Y MANEJO DE LA IN- TOXICACIÓN OCUPACIONAL}

El diagnóstico de intoxicación por mercurio en salud ocupacional, tal como hemos visto, se establece con criterios clínicos y ocupacionales entre los que destacan:

1. Antecedente de exposición a mercurio comprobada por mediciones ambientales. El valor de referencia, valor umbral límite (TLV), de la ACGIH para mercurio elemental y sus formas inorgánicas, es 0,025 $\mathrm{mg} / \mathrm{m} 3 / 8$ horas día/5 días semana ${ }^{(21,25)}$.

2. La intoxicación mercurial debería ser considerada cuando un trabajador expuesto presenta:

- Síndrome digestivo: presencia de sabor metálico, mal aliento, náuseas, vómitos y diarrea, todos ellos no patognomónicos, pero indiciarios.

- Síndrome neurológico: aquí lo característico es irritabilidad, tristeza, ansiedad, insomnio, sueño agitado, temor, debilidad muscular, pérdida de memoria, excesiva timidez, susceptibilidad emocional, hiperexcitabilidad o depresión. El trabajador puede llegar a presentar encefalitis y síndrome psicoorgánico crónico y definitivo, que ocasionalmente termina en demencia. Pero, el signo capital es el temblor intencional, tipo temblor cerebeloso, asociado a ataxia, adiadococinesia y marcha de tipo cerebelosa. Puede haber exageración de los reflejos patelares, pero no son frecuentes espasmos musculares ni parálisis flácida (5).

- Síndrome oftalmológico; aquí busquemos signos oculares precoces de intoxicación, como: escotomas anulares y centrales, además de visión tubular y nistagmus. En el examen con lámpara de hendidura, es posible encontrar el signo de Atkinson, reflejo parduzco bilateral y simétrico, en la cápsula anterior del cristalino, que no afecta la visión. Estudios actuales apoyan el daño en la visión de colores ${ }^{(12-14)}$.
- Síndrome renal: los signos y síntomas de glomérulonefrosis de aspecto semejante a la lipoide y en casos avanzados síntomas por glomerulonefritis proliferativa extracapilar, caracterizan a esta intoxicación.

3. Indicadores biológicos de exposición.

- Mercurio inorgánico total en orina: su valor no debe ser mayor de $35 \mu \mathrm{g} / \mathrm{g}$ creatinina, muestra tomada antes de la jornada laboral.

- Mercurio inorgánico total en sangre: menor a $15 \mu \mathrm{g} / \mathrm{L}$, en muestra tomada al final de la jornada diaria o al fin de semana laboral ${ }^{(21,22)}$.

- En exposición ocupacional, se recomienda medir mercurio en orina de 24 horas, mientras que en exposición aguda o accidental se debe preferir su dosaje en sangre ${ }^{(23,24)}$.

- Para valoración de la función renal, la vigilancia médica debe hacerse con indicadores precoces de daño renal, como la excreción de B 2 microglobulina, $\mathrm{N}$-acetil-D-glucosaminidasa (NAG) o $B$ galactosidasa $(5,25,29,30)$.

- Estos análisis de mercurio en orina y en sangre, y los dosajes enzimáticos son factibles de realizar en laboratorios toxicológicos especializados que existen en nuestra ciudad capital y en algunas capitales de departamento.

Realizado el diagnóstico y desde el punto de vista de salud ocupacional, la primera medida a tomar es alejar al trabajador de la exposición al mercurio, rotándolo a un puesto de labor limpio y, si esto no es posible, prescribir descanso médico en este lapso. Dos meses sin contacto con el mercurio basta para reducir su nivel en líquidos biológicos al 50\% del valor BEI.

El tratamiento usual debería ser con fármacos de última generación, como DMPS, el 2,3 dimercaptopropano-1-sulfonato o Dimanal $^{R}$ o con DMSA, el ácido 2,3 dimercaptosuccinico o Succimer $^{R}$. En la industria minera peruana usamos $\mathrm{D}$ - penicilamina o 2,3 - dimercaptopropanol, BAL.

Cuando el trabajador se cura del cuadro clínico, podría retomar su trabajo habitual solamente si los valores del tóxico en el ambiente de labor estén por debajo de lo permisible y el trabajador no tenga estigmas de exposición. Si los tuviera, y aún cuando el valor ambiental laboral esté bajo el TLV, se le apartará definitivamente y trabajará en un ambiente totalmente libre del tóxico ${ }^{(21,25)}$.

Como conclusiones, diremos que el mercurialismo es una enfermedad ocupacional grave. Aún no se dispone de parámetros bioquímicos que sirvan como indicadores de efecto y que a la vez permitan el control biológico, cuando las alteraciones orgánicas son todavía reversibles. El tratamiento del mercurialismo tiene eficacia limitada, pues establecido el daño es irreversible. Los compuestos inorgánicos del mercurio no son cancerígenos y no tienen los efectos teratógenos que sí poseen los compuestos orgánicos. Los niveles de mercurio total en sangre de $15 \mu \mathrm{g} / \mathrm{L}$ y en orina $35 \mu \mathrm{g} / \mathrm{g}$ creatinina han mostrado ser eficaces como indicadores biológicos, para evitar repercusiones negativas en la salud del trabajador expuesto; sin embargo, se debería profundizar la investigación de los efectos a estos niveles de exposición 'seguros', sobre la visión del trabajador.

\section{REFERENCIAS BIBLIOGRÁFICAS}

1. Zenz C, ed. Occupational medicine: principles and practical applications. 2nd Ed. Chicago: Mosby; 1988.

2. Español CS. Estudio sobre correlación de valores de mercurio en ambiente y fluidos biológicos con la aparición de sintomatología clínica en trabajadores de Minas de Almadén. Almadén: Minas de Almadén y Arrayanes S. A.; 1990.

3. Parmeggiani L, ed. Encyclopedia of Occupational Health and Safety. 3th Ed. Geneva: International Labor Office; 1989.

4. WHO. El Mercurio en el Sector de la Salud. Ginebra: OMS Departamento de Salud Pública y Medio Ambiente Agua, Saneamiento y Salud; 2004.

5. Ellemhorn MJ. Medical Toxicology. 2nd Edition. Baltimore: Williams Wilkins; 1996.

6. Casarett and Doull's. Toxicology. The Basic Science of Poison. 6th Edition. Barcelona: Editorial Mc Millan; 2001.

7. Friberg L, Nordberg GF, Vouk VB, eds. Handbook of toxicology of metals. Amsterdam: Elsevier; 1979.

8. Friberg LM, Piscator GF, Kjelistrom T. Cadmium in the Environment. 2nd Ed. Cleveland: CRC Press; 1974.

9. Chaparro E. La llamada pequeña minería: Un renovado enfoque empresarial, División de Recursos Naturales e Infraestructura. Santiago de Chile: CEPAL; 2001.

10. La Dou J. Medicina Laboral y Ambiental. $2^{\text {a }}$ Ed. México, DF: Manual Moderno; 1999.

11. Carroll L. Alicia en el país de las maravillas. Traductor Armiño M. Madrid: Ed Edad; 2002. p. 191.

12. Pavel UP, Gobba FM, Nerudová J, Luká E, Ábelková Z, Cikrt M. Color discrimination impairment in workers exposed to mercury vapor. Environ Toxicol Pharmacol. 2005;19(3):523-9.

13. Ventura DF, Costa MTV, Costa MF, Berezovsky A, Salomão SR, Simões AL, et al. Multifocal and 
full-field electroretinogram changes associated with color-vision loss in mercury vapor exposure. Environment Research. 1998;77(2):173-7.

14. Cavalleri A, Gobba F. Reversible color vision loss in occupational exposure to metallic mercury. Environmental Research. 1998;77(2):173-7.

15. Levy B S, Wegman D H, eds. Occupational Health. Boston: Little, Brown and Co.; 1988.

16. Eto K, Takizawa Y, Akagi H, Haraguchi K, Asano $\mathrm{S}$, Takahata N, Tokunaga H, Differential diagnosis between organic and inorganic mercury poisoning in human cases - the pathologic point of view. Toxicol Pathol. 1999;27(6):664-71.

17. Dabeka RW. Examen de mercurio total en alimentos de la dieta y valoración de la ingesta dietética del mercurio de los adultos y de niños en dos ciudades canadienses, 1998-2000. Rev Alimento Addit Contam. 2003;20(7):629-38.

18. Oken E, Kleinman KP. Estudio de consumo de pescado entre mujeres embarazadas. Rev Obstet Gynecol. 2003;102(2):346-51.

19. WHO. Committee on Toxicology Union Kingdom. Study about mercury on fishes and shellfish. England: The Commitee; 2003.

20. Ministerio de Energia y Minas del Perú. DS-0462001-EM. Lima: El Ministerio; 2001.

21. ACGIH, TLVs. BEls. Threshold limits values for chemical substances and physical agents.
Biological exposure indices. Cincinnati: ACGIH; 2007.

22. Ramírez AV. Biomarcadores en monitoreo de exposición a metales pesados en metalurgia. An Fac Med Lima. 2006;67(1):49-58.

23. Protrowski GK, Trojanowska D, Mogilnicka EM. Excretion kinetics and variability of urinary mercury in works exposed to mercury vapour. Int Arch Occ Environ Health. 1975;35:245.

24. Lovejoy HB, Bell ZB Jr. Mercury exposure evolution and their correlation with urine mercury excretions. J Occup Med. 1973;15:964.

25. Agency for Toxic Substances and Disease Registry. Mercury Chemical Summaries - Summary memorandum (of literature review). Atlanta: ATSDR; 2005.

26. Mant TGK. Clinical studies with Dimercaptopropane sulfonate in mercury poisoning. Hum Toxicol. 1987;4:346.

27. Olson KR, ed. Poisoning and drug overdose: A Lange Clinical Manual, 4th Ed. New York: Lange Medical Books/McGraw-Hill; 2004.

28. Shannon M, Graef J, Lovejoy FH. Efficacy and toxicity of $d$-penicillamine in low-level lead poisoning. J Pediatr. 1988;12:799-804.

29. Ramírez A V, Fiestas SE, Protocolo de Vigilancia en Salud Ocupacional. Riesgo químico mercurio. Lima: Pacifico Salud, Medica; c2005. Disponible en: http://www.minem.gob.pe/archivos/ dgm/publicaciones/expedientes/yanacocha/ justificacion/4.2-R3\%20Protocolo\%20de\%20Vigi lancia\%20en\%20Salud\%200cupacional\%20de\% 20Riesgo\%20Qu\%C3\%ADmico-Mercurio-2005. pdf

30. Magdo HS, Forman J, Graber N, Newman B, Klein $\mathrm{K}$ Satlin L, et al. Nephrotoxicity in young child exposed from contaminated well water. Environ Health Perspect. 2007;115(8):1237-41.

Manuscrito recibido el 26 de febrero de 2008 y aceptado para publicación el 15 de febrero de 2008.

\section{Correspondencia:}

Augusto V. Ramirez, MD.

Los Naranjos 139 B. El lingenio.

POB 110. Serpost.

Cajamarca, Perú.

Correo-e: augustovram@yahoo.es 